\title{
Species Identification and Antifungal Susceptibility Profile of Candida Isolates Obtained from Oral Lesions in Patients Attending Outpatient Department of Academic Dental Hospital
}

\author{
Ashwini Bhosale ${ }^{1 *}$, Pratibha Narang ${ }^{2}$ and Deepak Thamke ${ }^{2}$ \\ ${ }^{1}$ Department of Microbiology, Sinhagad Dental and Hospital, Pune, Maharashtra, India \\ ${ }^{2}$ Department of Microbiology, Mahatma Gandhi Institute of Medical Sciences, \\ Sewagram, Maharashtra, India \\ *Corresponding author
}

\begin{tabular}{l} 
Key w o r d s \\
$\begin{array}{l}\text { Antifungal } \\
\text { resistance, Candida } \\
\text { albicans, Oral } \\
\text { candidiasis }\end{array}$ \\
\hline Article Info \\
\hline $\begin{array}{l}\text { Accepted: } \\
\text { 14 December } 2018 \\
\text { Available Online: } \\
\text { 10 January } 2019\end{array}$ \\
\hline
\end{tabular}

\section{Keywords}

Antifungal albicans, Oral candidiasis

14 December 2018

Available Online:

10 January 2019

\section{Introduction}

The incidence of fungal infections has dramatically increased worldwide (RazzaghiAbyaneh et al., 2014). While HIV/AIDS has been an important predisposing factor for the rise, other conditions like malignancies, use of broad spectrum antibiotics, indwelling medical devices and diabetes have also contributed to the increase. Among various
Candida species is the only fungal pathogen that causes variety of afflictions that ranges from superficial mucosal infections to life-threatening disseminated mycoses. Oral candidiasis is a common fungal infection caused by an overgrowth or infection due to Candida spp. Candida albicans is considered as the primary etiology of various clinical types of candidiasis including oral lesions. However, in recent years research studies have highlighted the greater recognition of non-albicans Candida (NAC) spp. The present study was conducted with an aim to study species distribution and antifungal susceptibility profile of Candida isolates obtained from oral lesions. HIV infection, diabetes, dentures and malignancy were main predisposing factors. C. albicans $(79.8 \%)$ was the predominant isolate. NAC spp. were isolated from 21(20.2\%) cases. Fluconazole resistance was observed in $9.6 \%$ of $C$. albicans whereas $23.8 \%$ of NAC spp demonstrated resistance to fluconazole. From this study, it can be concluded that, although an epidemiological shift towards non albicans Candida species is noted in recent years, $C$. albicans still remains the pervasive pathogen. Antifungal susceptibility testing of Candida isolates is extremely important for selection of most appropriate therapeutic agent. 
2014). Fungi belonging to genus Candida are commensals and harmlessly colonize various niches of human body like the oral cavity, gastrointestinal tract, vagina and skin. Under certain circumstances, this "innocuous commensal" is transformed into a diseasecausing "parasitic" form. This transition is dependent on both host's predisposing factors and virulence of infecting strain (Deorukhkar et al., 2014).

Oral candidiasis is a common fungal infection caused by an overgrowth or infection due to Candida spp (Akpan and Morgan, 2002). The incidence of oral candidiasis varies as per age and certain predisposing factors. Although, oral candidiasis is rarely fatal, it often leads to local discomfort, dysphagia and alteration in sensation of taste that result in poor nutrition, slow recovery from illness and prolonged hospital stay (Akpan and Morgan, 2002). In most of the cases Candida albicans is considered as the primary etiological agent for various clinical types of candidiasis including oral lesions. However, in recent years research studies have highlighted the emergence of non-albicans Candida (NAC) spp like $C$. tropicalis, C. glabrata and C. krusei (Raju and Rajappa, 2011) which have different drug susceptibilities. Species identification of the isolates has therefore, become necessary for initiation of species-directed therapy.

The present study was conducted with an aim to study species distribution and antifungal susceptibility profile of Candida isolates obtained from oral lesions.

\section{Materials and Methods}

The present study is a part of $\mathrm{PhD}$ thesis in the Department of Microbiology, Mahatma Gandhi Institute of Medical Sciences (MGIMS), Sevagram in collaboration with Sinhagad Dental College and Hospital, Pune, Maharashtra, India. The protocol of study was approved by Institutional Ethics Committee. The study included OPD patients presenting with oral lesions. Informed consent was obtained from all participants.

A total of 2 oral swabs were collected from these patients. Out of these, one swab was used for preparation of smear for Gram staining whereas, other swab was inoculated on Sabouraud dextrose agar (SDA) slope. The SDA slope was incubated at $37^{\circ} \mathrm{C}$ for 7 days and observed daily for growth of Candida spp.

Candida spp. produces curdy white, opaque, flat, smooth and pale colored colonies with sweet smell similar to that of ripe apple (Lynch 1994). The Candida isolates were identified upto species level as per standard mycological protocol which included germ tube test, sugar fermentation and assimilation tests and growth pattern on CHROM agar Candida (Koneman et al., 1985).

The antifungal susceptibility testing was done by disc diffusion method and interpreted according to Clinical Laboratory Standards Institute's M44-A guidelines (CLSI, 2004). Isolates were tested for antifungal drugs like amphotericin B, fluconazole, ketoconazole and itraconazole. Antifungal discs were procured from Himedia Laboratories Pvt. Ltd Mumbai. Demographic and clinical features of patients were recorded and analyzed.

\section{Results and Discussion}

During the study period, a total of 460 patients with oral lesions attended the OPD of dental hospital. Out of these, $364(79.1 \%)$ were males and 96 (20.9\%) were female patients. The mean age of patients was 41 years (range: 20-75 years).

A total of $322(70 \%)$ patients were tobacco chewers, cigarette smoking was reported by 9 $(1.9 \%)$ patients. HIV infection, diabetes, 
dentures and malignancy were main predisposing factors. A total of 62 patients with oral lesions were positive for HIV infection. Oral carcinoma was the commonest malignancy seen. Poor oral hygiene was seen in $36(7.8 \%)$ cases.

Leukoplakia, pseudomembranous thrush and angular cheilitis were common oral lesions in patients. In HIV infected individuals, pseudomembranous thrush was the common clinical type of oral lesions.

Candida spp. were isolated from 104 (22.6\%) patients. The species wise distribution of Candida isolates is shown in figure 1. $C$. albicans was the predominant isolate 83 out of 104(79.8\%). NAC spp. were isolated from $21(20.2 \%)$ cases. They were $C$ glabrata $7, C$ tropicalis 7, C krusei 3 and $C$ gulliermondii 4 .

The antifungal susceptibility profile of Candida isolates is shown in Table 2. A total of $13(12.5 \%)$ isolates were resistant to fluconazole. Fluconazole resistance was observed in $9.6 \%$ of $C$. albicans whereas $23.8 \%$ of NAC spp demonstrated resistance to fluconazole. However, there was no statistical difference observed between fluconazole resistance between NAC spp. and $C$. albicans (Fischer's exact test, $P=0.13$ ). In the present study, a total of 3 Candida isolates were SDD (Susceptible dose dependent) to fluconazole.

Itraconazole resistance in $C$. albicans and NAC spp were $4.8 \%$ and $4.7 \%$ respectively. Ketoconazole resistance was observed only in C. albicans isolates. Amphotericin B resistance was noted in $7(8.4 \%) C$. albicans isolates. Among NAC spp. amphotericin B resistance was observed in only $C$. tropicalis isolates

Oral candidiasis is one of the most common clinical presentations of Candida reported by clinicians of different specialties worldwide
(Razzaghi-Abyaneh et al., 2014). Oral candidiasis presents in many forms. In the present study leukoplakia, pseudomembranous thrush and angular cheilitis were common oral lesions seen. Leukoplakia due to Candida spp. is characterized with white localized patches with irregular borders that are difficult to remove (Lopez-Martinez 2010). Pseudomembranous candidiasis is characterized by white membranes on the oral mucosa and tongue (Samaranayake and Nair, 1995). It is made up of necrotic material and desquamated epithelia invaded by yeast cells and hyphae (Samaranayake and Nair, 1995). In the current study, pseudomembranous thrush was most commonly seen in HIV infected patient. Similar observation was reported by Samaranayake and Nair (Samaranayake and Nair, 1995).

A variety of local and systemic factors are implicated for oral candidiasis. These include mechanical factors like ill-fitting dentures, short term factors like antibiotic therapy and factors related to immune and underlying disease status of the host. In the present study $13.5 \%$ of patients with oral lesions were HIV infected. Oral lesions are reported in about $64 \%$ of HIV/AIDS patients in India (Deorukhkar et al., 2012). Pseudomembranous candidiasis is the most common oral lesion reported in HIV infected individual (Coleman et al., 1997). It occurs in $17-43 \%$ cases with HIV infection and in more than $90 \%$ of AIDS patients (Deorukhkar et al., 2012).

In this study diabetes, dentures and malignancy were other risk factors associated with oral candidiasis. In diabetes, the presence of glucose enhances growth of Candida in saliva and its adherence to buccal epithelial cells (Akpan and Morgan 2002). Presences of dentures create a micro environment for Candida growth. Oral candidiasis occurs in as many as $65 \%$ of geriatric population wearing dentures (Akpan and Morgan 2002). 
Table.1 The lesion wise and species wise distribution of Candida isolate

\begin{tabular}{|l|c|c|c|c|c|}
\hline Type of lesion & C. albicans & C. tropicalis & C. glabrata & C. krusei & C. guilliermondii \\
\hline $\begin{array}{l}\text { Pseudomembranous } \\
\text { candidiasis (n=29) }\end{array}$ & 26 & 1 & 1 & 1 & - \\
\hline Angular chelitis (n=7) & 6 & 1 & - & - & - \\
\hline $\begin{array}{l}\text { Denture stomatitis } \\
\text { (n=6) }\end{array}$ & 4 & 1 & 1 & - & - \\
\hline $\begin{array}{l}\text { Erythematous } \\
\text { candidiasis (n=3) }\end{array}$ & 2 & - & - & - & 1 \\
\hline Leucoplakia (n=12) & 11 & 1 & - & - & - \\
\hline Tobacco pouch (n=7) & 5 & 1 & - & - & 1 \\
\hline Oral cancer (n=15) & 14 & - & - & - & 1 \\
\hline $\begin{array}{l}\text { Oral submucous } \\
\text { fibrosis (n=3) }\end{array}$ & 2 & 1 & & & \\
\hline Multiple lesion (n=22) & 13 & 1 & 5 & 2 & \\
\hline Total (n=104) & 83 & 7 & 7 & 3 & \\
\hline
\end{tabular}

Table.2 Antifungal susceptibility profile of Candida isolates

\begin{tabular}{|c|c|c|c|c|c|c|c|c|c|c|c|c|}
\hline \multirow[b]{2}{*}{ Candida spp } & \multicolumn{3}{|c|}{ Fluconazole } & \multicolumn{3}{|c|}{ Itraconazole } & \multicolumn{3}{|c|}{ Ketoconazole } & \multicolumn{3}{|c|}{ Amphotericin B } \\
\hline & $\mathrm{S}$ & SDD & $\mathrm{R}$ & $S$ & SDD & $\mathrm{R}$ & $\mathrm{S}$ & SDD & $\mathrm{R}$ & $\mathrm{S}$ & SDD & $\mathrm{R}$ \\
\hline $\begin{array}{l}\text { C. albicans } \\
\mathrm{n}=\mathbf{8 3}\end{array}$ & 74 & 1 & 8 & 79 & - & 4 & 74 & 2 & 7 & 74 & 2 & 7 \\
\hline $\begin{array}{l}\text { C. glabrata } \\
n=7\end{array}$ & 5 & 2 & - & 7 & - & - & 7 & - & - & 7 & - & - \\
\hline $\begin{array}{l}\text { C. tropicalis } \\
n=7\end{array}$ & 5 & - & 2 & 5 & 1 & 1 & 6 & 1 & - & 5 & 1 & 1 \\
\hline $\begin{array}{l}\text { C. } k r u s e i \\
\mathrm{n}=3\end{array}$ & 3 & - & - & 3 & - & - & 3 & - & - & 3 & - & - \\
\hline $\begin{array}{l}\text { C. guilliermondii } \\
\mathrm{n}=4\end{array}$ & 1 & - & 3 & 4 & - & - & 3 & 1 & - & 3 & 1 & - \\
\hline $\begin{array}{l}\text { Total } \\
n=104\end{array}$ & $\begin{array}{c}88 \\
(84.6 \\
\%)\end{array}$ & $\begin{array}{c}3 \\
(2.9 \%)\end{array}$ & $\begin{array}{l}13 \\
(12 . \\
5 \%)\end{array}$ & $\begin{array}{c}98 \\
(94.2 \\
\%)\end{array}$ & $\begin{array}{c}1 \\
(0.9 \\
\%)\end{array}$ & $\begin{array}{c}5 \\
(4.8 \\
\%)\end{array}$ & $\begin{array}{c}93 \\
(89 . \\
4 \%)\end{array}$ & $\begin{array}{c}4 \\
(3.8 \\
\%)\end{array}$ & $\begin{array}{c}7 \\
(6.7 \\
\%)\end{array}$ & $\begin{array}{c}92 \\
(88 . \\
4 \%)\end{array}$ & $\begin{array}{c}4 \\
(3.8 \\
\%)\end{array}$ & $\begin{array}{c}8 \\
(7.7 \\
\%)\end{array}$ \\
\hline
\end{tabular}


Fig.1 The species wise distribution of Candida isolates

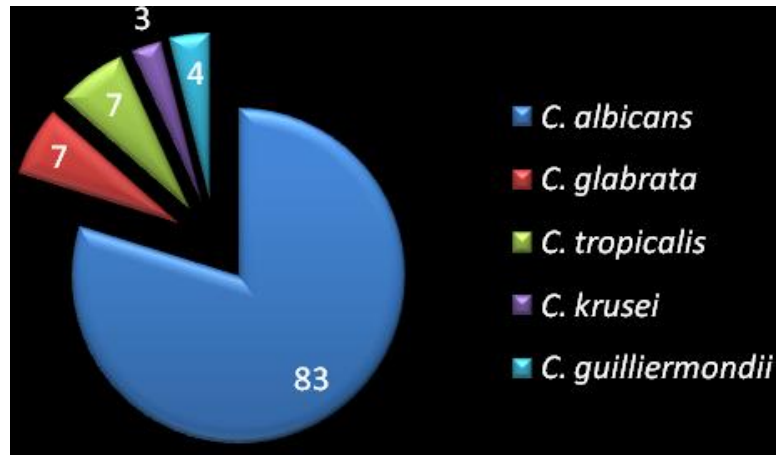

The relationship between Candidial leukoplakia and malignancy is well recognized. Oral neoplasias can be further complicated by Candida infection (Samaranayake and Nair 1995).

In this study, $C$. albicans was the predominant isolate from oral lesions. Our observation was similar to that of Shafi et al., (Shafi et al., 2015) and Mane et al., (Mane et al., 2010). However, in the study of Deorukhkar et al., (Deorukhkar et al., 2012) NAC spp. were predominant isolates. Species variation may be due to various reasons including host factors like diet, oral hygiene and long treatment with fluconazole and use of commercially available kit system for identification of Candida spp. In the present study disc diffusion method was used for screening of antifungal resistance in Candida Spp. As compared to CLSI broth microdilution method, disc diffusion technique is comparatively less cumbersome and less time consuming (Deorukhkar et al., 2012) and can be easily incorporated in laboratory for routine basis. As compared to amphotericin B and other azoles, Candida isolates demonstrated high resistance to fluconazole. Resistance to fluconazole is of concern because it is used as first line drug for prophylaxis and treatment of candidiasis (Dismukes, 2000). It has good bioavailability, high water solubility and long half-life. Additionally, it is easy to administer and is comparatively less toxic (Deorukhkar and Saini, 2014).

Fluconazole resistance in the present study, was observed in $9.6 \%$ of $C$. albicans whereas $23.8 \%$ of NAC spp demonstrated resistance to fluconazole. NAC spp. are either intrinsically resistant to fluconazole or may acquire resistance during course of therapy.

From this study, it can be concluded that oral candidiasis is one of the most common affliction of the oral cavity and though an epidemiological shift towards non albicans Candida species is noted in recent years, $C$. albicans still remains the pervasive pathogen. Antifungal susceptibility testing of Candida isolates should be carried out for selection of most appropriate therapeutic agent.

\section{References}

Akpan A and Morgan R 2002. Oral candidiasis. Postgrad Med J. 78:455459. 
Coleman D, Sullivan D, Bennett D, Moran G, Barry H and Shanley D 1997. Candidiasis: the emergence of a novel species, Candida dubliniensis. AIDS. 11: 557-567.

Deorukhkar S, Katiyar R and Saini S 2012. Species identification and antifungal susceptibility pattern of Candida isolates from oropharyngeal lesions of HIV infected patients. Natl J Integr Res Med. 3:86-89.

Deorukhkar S, Saini S and Mathew S 2014. Non-albicans Candida Infection: An Emerging Threat. Interdis Perspect Infect Dis. Volume 2014, Article ID 615958, 7 Pp. doi: $10.1155 / 2014 / 615958$.

Dismukes W 2000. Introduction to antifungal drugs. Clin Infect Dis. 30: 653-702.

Koneman E, Robberts G 1985. Practical Laboratory Mycology. $3^{\text {rd }}$ edn. Williams and Wilkins, Baltimore: 143-163.

Lopez-Martinez R 2010. Candidosis, a new challenge. Clinics in Dermatol. 28:178184. Doi: $10.54027 / 2011 / 487921$.

Lynch D 1994. Oral candidiasis: History, classification and clinical presentation. Oral Surg Oral Pathol Oral radiol Endod. 78:189-193.
Mane A, Panchvalli, Bembalkar S, Risbud A 2010. Species distribution and antifungal susceptibility of oral Candida colonizing or infecting HIV infected individuals. Indian J Med Res. 131:836838.

Raju S and Rajappa S 2011. Isolation and identification of Candida from the oral cavity. International Scholarly Network. Volume 2011, Article ID 487921, 7 Pp.

Razzaghi-Abyaneh M, Sadeghi G, Zeinali E, Alirezaee M, Shams-Ghahfarokhi M, Amani A, et al.,. 2014. Species distribution and antifungal susceptibility of Candida spp. isolated from superficial candidiasis in outpatients in Iran. J Mycol Med. 24: e43-e50.

Samaranayake L, Nair R. 1995. Oral Candida infection-A review. Indian J Dent Res. 6:69-82.

Seneviratne C, Jin L, Samaranayake L. 2008. Biofilm lifestyle of Candida: a mini review. Oral Dis. 14:582-590.

Shafi F, Padmaraj S, Mullessery N. 2015. Species distribution and antifungal susceptibility pattern of Candida causing oral candidiasis among hospitalized patients Arch Med Health Sci., 3: 247-251.

\section{How to cite this article:}

Ashwini Bhosale, Pratibha Narang and Deepak Thamke. 2019. Species Identification and Antifungal Susceptibility Profile of Candida Isolates Obtained from Oral Lesions in Patients Attending Outpatient Department of Academic Dental Hospital. Int.J.Curr.Microbiol.App.Sci. 8(01): 1912-1917. doi: https://doi.org/10.20546/ijcmas.2019.801.201 